\title{
Research of the echo-impulse method of control of drilled pieces (non-destructive control)
}

\author{
Dmitry Snezhkov ${ }^{1}$, Neli Budrevich ${ }^{1}$ \\ ${ }^{1}$ Belarussian National Technical University, 220013
}

\begin{abstract}
The results of field and computational experimental studies of the pulse echo method are presented. Typical reflectograms of bored piles are presented. The factors that complicate the interpretation of control data are noted. An analysis of the formation of the fronts of an acoustic pulse during its shock excitation is presented. Estimates of the influence of reinforcement on the estimates of the propagation velocity of an acoustic pulse are given.
\end{abstract}

\section{Introduction}

Acoustic methods currently have practically no alternative when monitoring the integrity of large-sized and extended reinforced concrete products in cases where access to the product is limited, for example, foundation elements, piles, etc. A typical method for controlling the continuity and length of reinforced concrete piles is the vibration-acoustic or its western version, the pulse-echo method (Puls Echo Metod PEM). The tests are carried out by forming an acoustic wave impulse and recording its response from inhomogeneities in the pile body. The main information parameter is the time interval between the moment of the acoustic pulse initiation and the moment of recording the reflected acoustic signals. As additional parameters, one can consider the amplitude and frequency indicators of the measured vibrational accelerations and velocities at the control points during the passage of an acoustic wave. The approximate value of the acoustic wave propagation velocity in the concrete of the pile can be determined by carrying out preliminary calibration tests on the piles stored at the construction site, according to their known length and the measured time of the appearance of the acoustic response, which is possible only for driven piles at the stage of object erection. When inspecting the driven or manufactured bored piles installed in the design position, you can use either the ultrasonic pulse method according to state standard 17624-2012 [1], or the vibration-acoustic method in relation to the cross-section of the protruding part of the pile.

Despite the fact that the use of these methods for controlling reinforced concrete piles has ceased to be a rarity, the level of their methodological regulation leaves much to be desired. The main problem is the difficulty of unambiguous interpretation of the received echo signal. The available recommendations for the application of the pulse echo method are guided by the American ASTM D5882 - 16: "Standard Test Method for Low Strain Impact Integrity Testing of Deep Foundations". This standard specifies the minimum requirements for a pile testing procedure by applying a low impact load. The data obtained allow us to estimate the length, integrity and continuity of the pile, as well as the strength characteristics of the 
concrete of the pile, although the estimate is approximate. It should be borne in mind that acoustic test methods do not directly provide information on the bearing capacity of the pile, although this property should indirectly manifest itself in the decay rate of the echo pulse. The purpose of this article is to analyze the test methodology and the main factors that prevent an accurate and unambiguous interpretation of the results of identifying the heterogeneity of bored piles along the length.

\section{Measurement technique}

Reflection of a wave from the interface occurs due to a change in the total acoustic impedance of the pile. The acoustic impedance $\mathrm{Z}$ for a rod made of a homogeneous material is defined as:

$$
Z=S \cdot \sqrt{E \cdot \rho}
$$

where $S$ is the cross-sectional area of the pile, $E$ is the modulus of elasticity, $\rho$ is the density of the pile material.

Any change in $S, E$ or $\rho$ will lead to a change in impedance and, as a result, to the reflection of the acoustic wave. Reflections can be caused by the following reasons: the end of the pile; decrease / increase in cross-section; crack; change in the density of concrete; contrasting change of soil layers along the depth of the pile location. Defects do not cause reflection: gradual change in pile diameter; curved pile shape; minor defects. The sign of the reflection depends on the sign of the change in impedance at the interface between the media (Fig. 1). If the impedance value has decreased (decrease in the cross-section, deterioration of concrete quality), then the reflected pulse will coincide in sign with the shock one, and if it increased (increase in the cross-section, improvement in the quality of concrete), then the amplitudes will be of opposite signs. The pile length $H$, at a known wave propagation speed $V$, is calculated using the well-known formula (2):

$$
H=\frac{V \cdot t}{2}
$$

The parameters in formula (1) should be considered as given, taking into account the connection of the pile body with the surrounding soil, the physical and mechanical characteristics of the layers of which may differ significantly. Therefore, for greater reliability of the control results, it is important to take into account the soil profile and the technology of pile erection. Usually, these factors are ignored when analyzing the echo, or are considered only at a qualitative level.
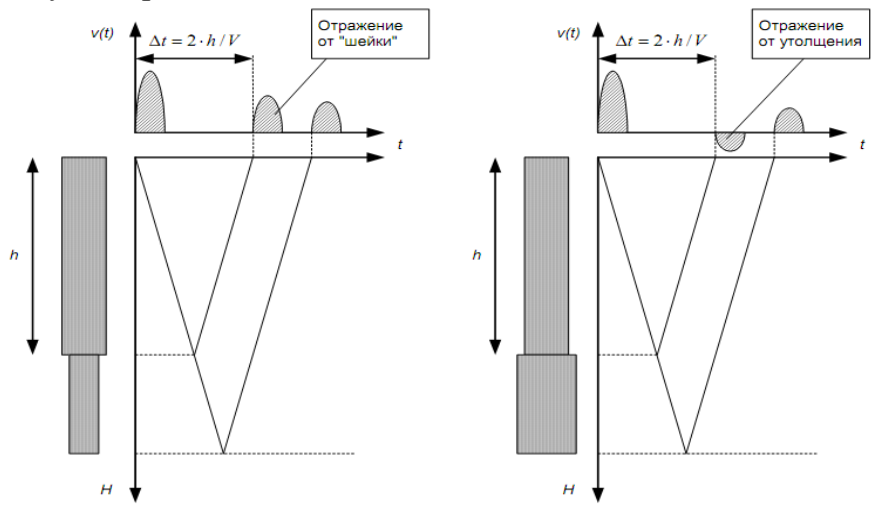

Fig. 1. Influence of the pile shape on the acoustic response shape [6] 
The quantitative calculation can be based on computer finite element models. In fig. 2 shows a reflectogram of a bored pile about $20 \mathrm{~m}$ long and $0.85 \mathrm{~m}$ in diameter. The initiating blow was made with a standard hammer with an "elastic" replaceable striker of the "Spectr-3" device.

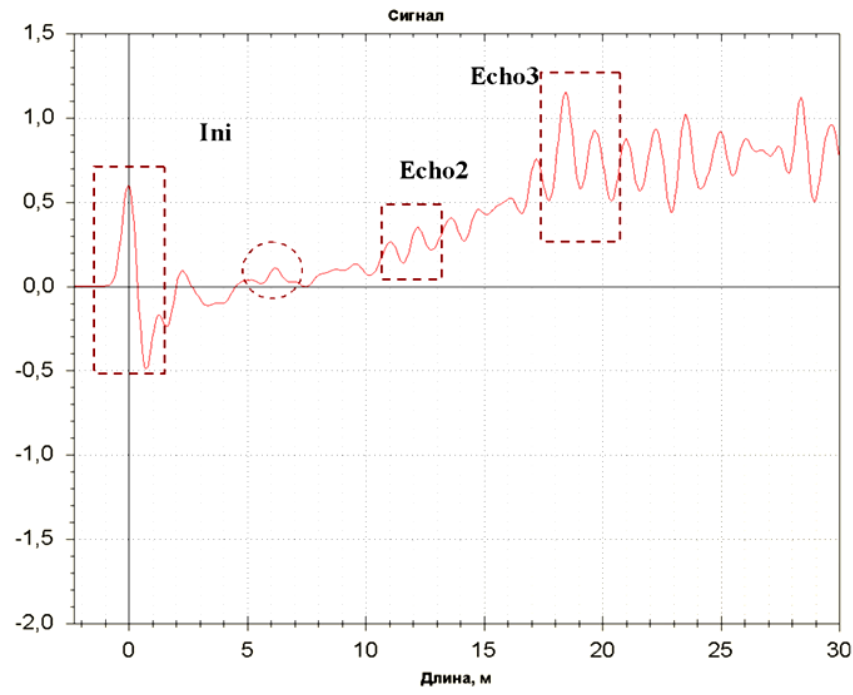

Fig. 2. Reflectogram of pile \# 2: Ini - initiating impulse (impact); Echo2, Echo3 - echo impulses

The Echo3 pulse corresponds to the reflection from the pile heel. The Echo2 pulse could not be uniquely identified. There is an impulse in Fig. 2 - it is circled, the appearance of which is consistent with the depth recorded during excavation work with a contrasting change in geological layers. There is no such information for the Echo2 pulse.

Increasing the information content and reliability of pile control, a positive effect can be obtained by the combined use of acoustic and radio wave methods. The soil profile according to $[2,3]$ is recorded in contrast. The speed of propagation of a radio wave depends on the electrophysical properties of the soil. Therefore, the GPR requires a calibration setting with reference to known marks that can be determined acoustically. Therefore, it is obvious that the combined use of a set of acoustic and electromagnetic methods can improve the reliability and accuracy of solving the problem of determining the length and continuity of concrete and reinforced concrete piles.

Another problem in the use of the pulse echo method is the complexity of the selection of information components of the acoustic signal against the background of the presence of numerous interferences. Most of them are due to standing waves within the cross-section of the pile, in particular, a standing shear wave arising after a point impact. Pile outlets can also cause strong quasi-harmonic interference masking the useful echo.

When monitoring free-lying piles and beams, there are usually no difficulties in analyzing the acoustic signal: the reflected echo signal practically does not experience attenuation, its amplitude at the registration point is close to the level of the initiating pulse. In Figure 3 (reinforced concrete beam $12 \mathrm{~m}$ long), three successively received echoes are clearly visible, the amplitude of which for each of 6 passes in total, the path was $72 \mathrm{~m}$, decreased by only $30 \%$. 


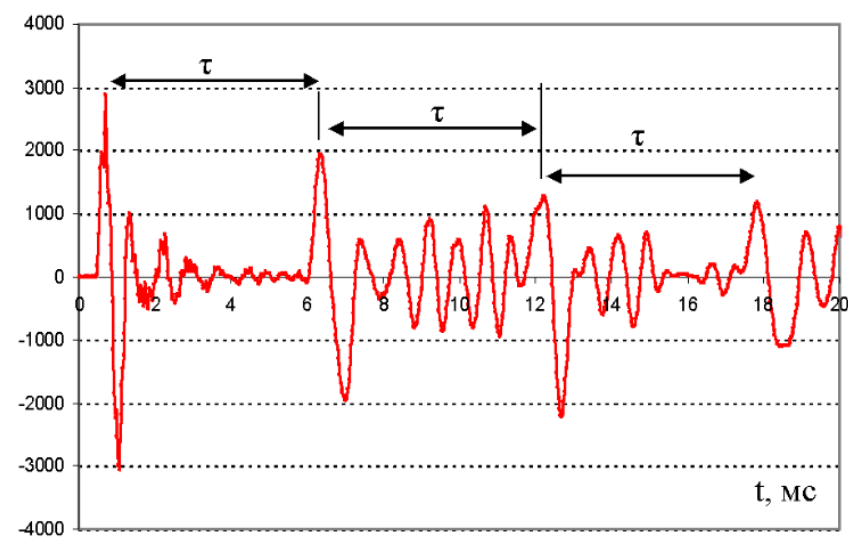

Fig. 3. Echo pattern for a reinforced concrete beam 12 meters long: maximum linear cross-sectional dimension $0.65 \mathrm{~m}$

The echo delay $\tau$ was $5.9 \mathrm{~ms}$. The longitudinal wave velocity $V_{l}=4380 \mathrm{~m} / \mathrm{s}$, determined by the ultrasonic pulse method. The dynamic Poisson's ratio $v$ is taken equal to $0.21[5,6]$. The period of the initiating pulse (see Fig. 3) is about $1.2 \mathrm{~ms}$, which corresponds to a frequency of $\sim 830 \mathrm{~Hz}$. The conditions for the propagation of a longitudinal wave along the beam correspond to the bar model. Based on this, we calculate the "pivot" velocity $V_{S}$ from the ratio

$$
\begin{gathered}
V_{l}^{2}=V_{S}^{2} \frac{(1-v)}{(1+v) \cdot(1-2 v)} \\
V_{S}^{2}=V_{l}^{2} \frac{(1-2 v) \cdot(1+v)}{(1-v)}=4380 \cdot \frac{0.58 \cdot 1.21}{0.78}=4380 \cdot 0.90=3942
\end{gathered}
$$

The calculated length of the beam was $L=3942 \cdot 5.8 \cdot 10^{-3} \cdot 0.5=11.63 \mathrm{~m}$. The calculation error was $-3.3 \%$. No noticeable increase in the duration of the probe pulse due to the manifestation of dispersion effects was recorded.

The tight contact of the lateral surface of the pile with the ground causes the energy of the acoustic signal to dissipate as it travels along the pile. To compensate for this attenuation, the signal is amplified. It is recommended that the signal amplification be made adaptive to the distance traveled or the delay time, assuming the attenuation coefficient $B$ to be constant. For comparison, Figure 4 shows the original echo signal and the signal after amplification with the gain $k$ depending on the delay time $t(\mathrm{~ms})$,

$$
k=e^{B \cdot t}
$$






Fig. 4. Timing diagrams (reflectograms) of the echo signal: pile with a cross section of $0.3 \times 0.3 \mathrm{~m}$, length $\sim 6 \mathrm{~m} ; 1-$ no amplification; 2 - with amplification $(B=1.152)$

It is obvious that the attenuation index $B$ depends on the technology of the pile manufacturing, its diameter, as well as on the characteristics of the soil and the conditions of interaction of the lateral surface of the pile with the soil. Figure 5 shows a reflectogram of a bored pile $0.85 \mathrm{~m}$ in diameter, approximate length $L \approx 21 \mathrm{~m}$.



Fig. 5. Timing diagrams (reflectograms) of the echo signal: pile $0.85 \mathrm{~m}$ in diameter, length $\sim 21 \mathrm{~m}$; signal gain with $\mathrm{B}=0.703$

A characteristic of the diagram (Fig. 5) is the appearance in the amplified acoustic signal of a noise (stochastic) component, commensurate with the useful echo signal from a level of - $24 \mathrm{~m}$ or more, which can be considered as the technical limit of the control system when determining the length of a pile of this type and diameter in the given geological conditions. The presence of a negative splash at the $6.5 \mathrm{~m}$ mark may indicate a contrasting change in the geological layers of the soil, or an increase in the pile section.

\section{Simulation of the process of propagation of an acoustic pulse wave in concrete mass with reinforcement}

The development of numerical modeling methods can help to improve the accuracy and reliability of data interpretation. The accuracy of determining the length and location of places of discontinuity of reinforced concrete piles is determined, first of all, by the accuracy 
of estimating the speed of propagation of an acoustic pulse in the body of the pile. The presence of a steel reinforcing cage is a factor that introduces uncertainty in the estimate of the speed of the acoustic pulse reflected from the irregularities and the pile heel, since the speed of the longitudinal acoustic wave in steel is about 1.5 times higher than that in homogeneous concrete. The existing guidelines for the use of the pulse echo method do not address this circumstance. In addition, with a point impact excitation of an acoustic pulse, accompanying "parasitic" acoustic waves appear in the body of the pile, significantly complicating the recorded signal for analysis.

To clarify the picture of the propagation of an acoustic pulse in reinforced concrete, a virtual sample was investigated - a finite element axisymmetric model, which is a cylinder $400 \mathrm{~mm}$ long and $150 \mathrm{~mm}$ in radius. Model material parameters: $E_{2}=20 \mathrm{GPa} ; v_{2}=0.2$; $\rho_{2}=2400 \mathrm{~kg} / \mathrm{m}^{3}$.

The task of modeling was to clarify the picture of the formation of the wave process and determine the degree of influence of reinforcement on the assessment of the propagation speed of an acoustic pulse in a reinforced concrete specimen. The position of the axis of the steel bar is taken to coincide with the axis of the cylindrical concrete mass of the model. The wave propagation medium was considered as a medium without damping; therefore, the amplitude (energy) ratios of the pulse propagation fronts are determined exclusively by geometric effects and the characteristic impedance of the rod material and its surrounding matrix [4]. For the purpose of "contrasting" display of the wave front, the acoustic pulse is initiated by a short-duration force action, its duration $T_{i}$ is taken equal to $7 \mu \mathrm{s}$, which is significantly less than the characteristic period $\tau$ of oscillations of a sample with a length of $0.4 \mathrm{~m}$, that is

$$
\tau=\frac{L}{V_{l}}=\frac{0,4}{5900}=68_{-} \text {мкс }
$$

The amplitude of the impulse of the force action is taken as $1 \mathrm{~N}$, the characteristics of the rod material are taken as typical: $\rho_{1}=7800 \mathrm{~kg} / \mathrm{m}^{3} ; E_{1}=260 \mathrm{GPa} ; v_{1}=0.3$; for which the "pivot" velocity of the acoustic pulse $V_{1}$

$$
V_{1}=\sqrt{\frac{E_{1}}{\rho_{1}}}=5774 m \cdot \mathrm{s}^{-1}
$$

The propagation velocity of the bulk longitudinal wave of the "concrete cylinder" $V_{2}$ is determined by the ratio

$$
V_{2}=\sqrt{\frac{E_{2}(1-v)}{\rho_{2}(1+v) \cdot(1-2 v)}}=3110 m \cdot s^{-1}
$$

and the velocity of propagation of the "rod" longitudinal wave $V_{2, S}$ at the accepted density of the concrete cylinder $\rho_{2}=2300 \mathrm{~kg} / \mathrm{m}^{3}$.

$$
V_{2, S}=\sqrt{\frac{E_{2}}{\rho_{2}}}=2950 \mathrm{~m} \cdot \mathrm{s}^{-1}
$$

Up to a propagation distance of the order of $2 \ldots 3$ diameters of a concrete sample, an acoustic wave can be considered volumetric for any pulse duration and recording the propagation time 
at the minimum threshold of the leading edge. At larger distances, frequency dispersion will manifest itself, which is expressed in an asymptotic decrease in the propagation velocity of the low-frequency components of the acoustic pulse wave from $3110 \mathrm{~ms}^{-1}$, corresponding to the body wave, to the "core" velocity of $2950 \mathrm{~ms}^{-1}$. The mode of excitation and registration of the impulse in the considered model is taken as the limiting one - the action of the impulse of the force $P(T)$ directly along the end of the reinforcing bar. The presence of a steel rod introduces significant changes in the nature of the propagation of the wave impulse. Since the pulse velocity in the rod is more than one and a half times higher than the pulse velocity in the concrete mass, the wavefront will be a cone moving along the $Z$ axis at a speed of about $5800 \mathrm{~m} / \mathrm{s}$. In this case, in the normal direction, the speed $V_{L, C}$ of the pulse front will coincide with the speed of the bulk wave in concrete, that is, $3110 \mathrm{~m} \cdot \mathrm{s}-1$.

In Figures 6 and 7, according to FE modeling data, the positions of the fronts of deformation pulses in a reinforced concrete specimen are displayed at different times $-17 \mu$ s and $49 \mu \mathrm{s}$. The rod diameter is $8 \mathrm{~mm}$. The dashed line in Figures 6 and 7 denotes the calculated position of the spherical front of the deformation pulse corresponding to the simulated sample without reinforcement. Since the top of the cone propagating along the axis of the reinforcement has a small radius of curvature (commensurate with the diameter of the reinforcing bar), then in this zone of the front there is a rapid decrease in the vibration intensity due to the transfer of wave energy in the reinforcing bar to two wave components leaving the sample mass.



Fig. 6. Spatial distribution of the displacement of the nodes of the FE model of the reinforced concrete sample at the time $17 \mu \mathrm{s}$ after the initiating pulse: $V_{L, C}, V_{L, A}-$ vectors of the propagation velocity of the pulse fronts of longitudinal waves in concrete and reinforcing bar

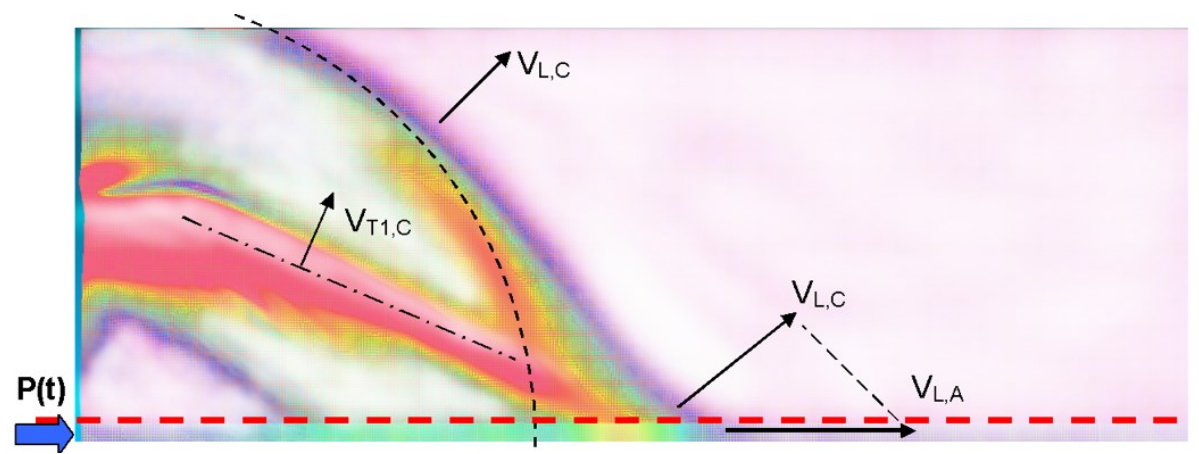

Fig. 7. Spatial distribution of the displacement of the nodes of the FE model of the reinforced concrete sample at the time $49 \mu$ s after the initiating pulse

One component of the wave in concrete is represented by the front of the longitudinal wave $V_{L, C}$, the second - by the front of the transverse wave $V_{T 1, C}$ (dash-dotted line in Fig. 7). In the zone of the cone, dispersion of the front is observed, its gradual "blurring" within the boundaries of the cone and the sphere. In fig. 4.8 shows the position of the pulse fronts at the time moment $(62 \mu \mathrm{s})$, when there is a noticeable weakening of the "leading" front-cone in the reinforcement, and the transfer of most of the vibrational energy to the wave in the concrete mass. The front of the shear wave $V_{T 1, C}$ is visible, which continues to "accompany" together with the front $V_{L, C}$ the top of the wave cone in the armature, and the intensity of which near the top of the cone also noticeably decreased. A new front of a transverse wave 
appeared, propagating with a velocity $V_{T 2}$ and forming at the point of reemission $\mathrm{s}$ on the lateral surface of the cylindrical model sample.



Fig. 8. Spatial distribution of the displacement of the nodes of the FE model of the reinforced concrete sample at the time $62 \mu \mathrm{s}$ after the initiating pulse: $V_{L, C}, V_{T 1, C}, V_{T 2, C}$, are the velocity vectors of the pulse fronts of the longitudinal and transverse waves

By the moment of time $75 \ldots 80 \mu$ s (see Fig. 9) only spherical fronts of the concrete mass manifest themselves, the wave in the steel rod has expended its energy.



Fig. 9. Spatial distribution of the displacement of the nodes of the FE model of the reinforced concrete sample at the time $77 \mu$ s after the initiating pulse

Along the circumference of the lateral surface of the sample-model crossing point $\mathrm{s}$, the transformation of the front of the longitudinal wave pulse into the front of the shear wave pulse with the vector of the front propagation velocity $V_{T 2, C}$ continues. In the initial stage of the formation of the front $V_{T 2, C}$, when point s was in the extreme left position, its velocity significantly exceeded the velocity of the front of the longitudinal wave, and the surface of the front $V_{T 2, C}$ near the end of the sample was coaxial with its side walls. As the front of the longitudinal wave propagates, the speed of movement of the point $s$ in the direction of the sample axis slows down and approaches the rod velocity of the longitudinal wave. In this case, there is a slight decrease in the intensity of the front $V_{T 2, C}$, and at a large distance from the point of oscillation initiation, when the spherical front of the longitudinal wave $V_{L, C}$ degenerates into a flat one, and the surface of the front $V_{T 2}$ degenerates into a cone with apex directed towards the end of the sample. This front will accompany the front of the longitudinal wave, keeping the intensity directly proportional to the intensity of the front of the longitudinal wave. Also in Figure 9, the front $V_{T 3, C}$ became noticeable. The analysis of the polarization of the oscillations of this front was not carried out, but its location at an angle of $30^{\circ} \ldots 40^{\circ}$ with respect to the normal to the end of the sample is consistent with the typical directional diagram (the maximum of the diagram corresponds to an angle of $\sim 37^{\circ}$ ) of the lateral lobe of the transverse wave with the considered point method of initiation of 
oscillations in the sample. This front, re-reflected from the walls of the cylindrical specimen, will also accompany the "leading" plane front of the longitudinal wave along the entire path of its propagation, gradually lagging behind it.

The above qualitative analysis of the formation of a wave pattern in a cylindrical sample indicates that the pattern of formation of the acoustic response of an extended element looks much more complicated than it is usually shown in manuals for devices of the shock-pulse method. In particular, the near-end region of the front $V_{T 2, C}$ can form in this region of the sample a damped transverse standing wave with a cylindrical front, the oscillations of which will be recorded immediately after the end of the initiating pulse by a sensor installed on the surface of the end. Figure 10 shows a reflectogram of a bored pile $0.85 \mathrm{~m}$ in diameter. The wavelength of oscillations accompanying the initiating pulse is $1.8 \ldots 1.9 \mathrm{~m}$, which corresponds to a half-wave standing shear wave in the area of the pile head. The oscillatory process is represented by $4 . . .5$ oscillations, which increase the "blind" zone of the method up to $5 . .66$ meters.

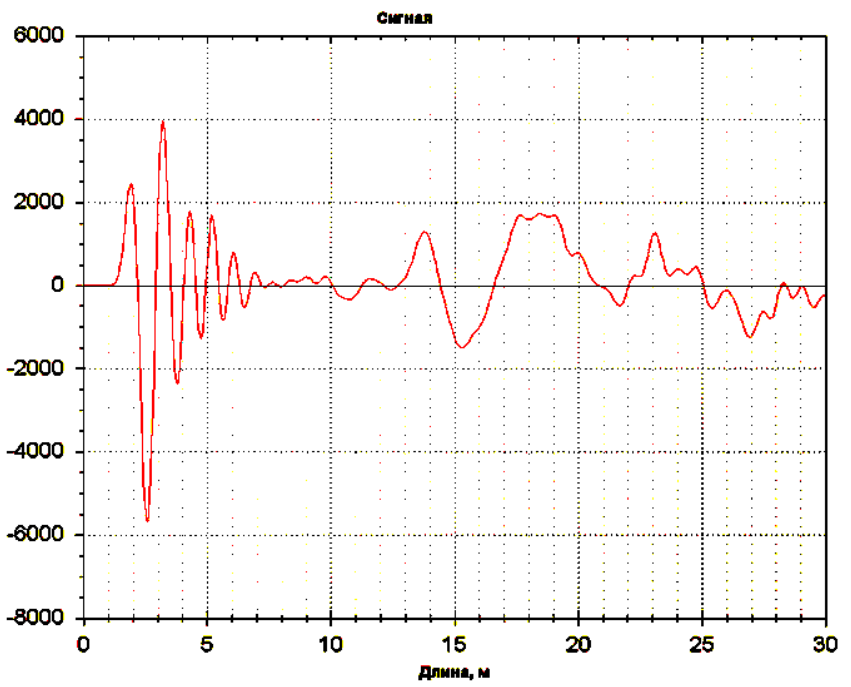

Fig. 10. Reflectogram of a bored pile $0.85 \mathrm{~m}$ in diameter and $12 \mathrm{~m}$ long

\section{Conclusion}

A method is proposed for calculating the boundaries of the influence of reinforcement when determining the time (speed) of propagation of an acoustic pulse in extended reinforced concrete elements, using computer simulation of acoustic processes. In particular, at distances exceeding $0.3 \mathrm{~m}$ for a rod diameter of $8 \mathrm{~mm}$, and $0.4 \ldots 0.6 \mathrm{~m}$ for a rod with a diameter of $16 \mathrm{~mm}$, there is no need to take into account the reinforcement. This conclusion is important for the method for determining the length and integrity of reinforced concrete piles by the pulse echo method (Puls Echo Metod PEM). With typical pile cross-sections of the order of $0.3 \times 0.3 \mathrm{~m}$ or more, lengths of more than $1 \ldots .2 \mathrm{~m}$, and typical reinforcement, the velocity of impulse propagation along the pile body should be defined as the "core" velocity of the longitudinal wave in concrete, without taking into account the presence of reinforcement.

The results of FE modeling indicate that with typical cross-sections and the degree of saturation with reinforcement, it manifests itself only in the initial stage of wavefront formation at distances of the order of $0.15 \ldots 0.5 \mathrm{~m}$ from the excitation point at the end. The 
contact between the lateral surface of the pile and the soil has a significantly greater effect on the conditions for the passage of the acoustic pulse along the body of the pile. If for a free beam (see Fig. 3) with a length of 12 meters, the attenuation index is $B \approx 0$, then for a 6 meter driven pile with a section of $0.3 \times 0.3 \mathrm{~m}$ in the soil (see Fig. 4) this indicator is 1.152 , which in absolute terms corresponds to 32-fold additional signal amplification from the $6 \mathrm{~m}$ mark in depth. For a bored pile with a diameter of $0.85 \mathrm{~m}$ (see Fig. 5), $B=0.703$, which for a signal from an elevation of $21 \mathrm{~m}$ in depth corresponds to a gain $k=1130$ in absolute units. With the ratio of the cross-sectional area of the longitudinal reinforcement to the crosssectional area of the pile within $1 / 200 \ldots 1 / 350$, taking into account the influence of reinforcement when controlling the continuity (determining the length) of piles by the shockpulse method, can be performed by introducing a negative correction within $0.3 \ldots 0.6 \mathrm{~m}$. This amendment is within the uncertainty of estimating the length of the pile, so its introduction is justified when controlling piles of small length of the order of $4 \ldots 6 \mathrm{~m}$. With typical pile cross-sections of the order of $0.3 \times 0.3 \mathrm{~m}$ or more, a length of more than $2 \ldots 3 \mathrm{~m}$, and typical reinforcement, the pulse propagation velocity along the pile body should be defined as the "core" velocity of the longitudinal wave in concrete, without taking into account reinforcement.

A factor that seriously complicates the interpretation of the echo signal and leads to large errors is the contrasting change of the geological layers of the soil. To identify the soil profile and take into account its influence on the results of acoustic tests, the joint use of acoustic and electromagnetic methods (georadars) is seen to be effective. Quantitative calculation of the influence of the geological factor can be based on computer finite element models.

\section{References}

1. State standard 17624-2012. Concrete. Ultrasonic method for determining strength. Moscow: Research, Design and Technological Institute of Concrete and Reinforced Concrete named after A. A. Gvozdev (NIIZHB), 2014. - 16 p.

2. V.V. Kapustin. Methods for studying the features of the propagation of acoustic waves in concrete piles using numerical modeling methods. Bulletin of Moscow University, Series 4, Geology, 3 - 2008 - pp. 65-70.

3. V.V. Kapustin. Application of seismic and acoustic technologies in the study of the state of underground building structures: Seismic technologies - 2008. - No. 1. - pp. 91-99.

4. D. Yu. Snezhkov, S. N. Leonovich, Yu. I. Lagun. On ultrasonic control of concrete strength and effects of reinforcement. Construction science and engineering: scientific and technical journal. - 2009. - No. 3. - P. 46-52.

5. D. U. Snezhkov, S. N. Leonovich. Multi-Wave Ultrasonic Control of Concrete. Science and technology. - 2017. - No. 4. - pp. 289-297.

6. D. Yu. Snezhkov, S. N. Leonovich. Multi-wave ultrasonic control of concrete. Vestnik Povolzhskogo gosudarstvennogo tekhnologicheskogo universiteta. Series: Materials. Structures. Technologies. 2017. No. 1. pp. 13-21. 